\title{
Gastrodin protects against LPS-induced acute lung injury by activating Nrf2 signaling pathway
}

\author{
Zhuo Zhang ${ }^{1}$, Jie Zhou ${ }^{2}$, Daqiang Song ${ }^{1}$, Yuhong Sun ${ }^{1}$, Changli Liao ${ }^{3}$ and Xian Jiang ${ }^{4}$ \\ 'Laboratory of Pharmacology, College of Pharmacy, Southwest Medical University, Luzhou, Sichuan, China \\ ${ }^{2}$ Laboratory of Science of Chinese Pharmacology, College of Pharmacy, Southwest Medical University, Luzhou, Sichuan, China \\ ${ }^{3}$ Research Department, College of Pharmacy, Southwest Medical University, Luzhou, Sichuan, China \\ ${ }^{4}$ Department of Anesthesiology, The Affiliated Hospital of College of Pharmacy, Southwest Medical University, Luzhou, \\ Sichuan, China
}

Correspondence to: Xian Jiang, email: jiangxian1716@163.com

Keywords: gastrodin, LPS, Nrf2, lung injury

Received: November 01,2016 Accepted: February 10,2017 Published: March 31, 2017

Copyright: Zhang et al. This is an open-access article distributed under the terms of the Creative Commons Attribution License (CC-BY), which permits unrestricted use, distribution, and reproduction in any medium, provided the original author and source are credited.

\section{ABSTRACT}

\begin{abstract}
Gastrodin (GAS), a phenolic glucoside derived from Gastrodiaelata Blume, has been reported to have anti-inflammatory effect. The aim of this study was to investigate the effects of GAS on LPS-induced acute lung injury in mice. ALI was induced by the intranasal administration of LPS and GAS was given $1 \mathrm{~h}$ or $12 \mathrm{~h}$ after LPS treatment. The results indicated that GAS treatment markedly attenuated the damage of lung injury induced by LPS. GAS attenuated the activity of myeloperoxidase (MPO) and downregulated the levels of pro-inflammatory cytokines TNF- $\alpha$, IL-6 and IL-1 $\beta$ in BALF. LPSinduced lung edema and lung function were also reversed by GAS. Furthermore, GAS was found to inhibit LPS-induced inflammatory cells infiltration. In addition, treatment of GAS inhibited LPS-induced NF-кB activation and up-regulated the expression of Nrf2 and HO-1. In conclusion, our results indicated that GAS had anti-inflammatory effects on LPS-induced acute lung injury. The anti-inflammatory mechanism of GAS was through the inhibition of NF-кB and activation of Nrf2 signaling pathways.
\end{abstract}

\section{INTRODUCTION}

Acute lung injury (ALI), the inflammation of the lung tissue, is a highly prevalent disease [1]. It is characterized by hypoxemia and pulmonary edema in lung [2]. This leads to a significant cause of mortality in critically ill patients. Recent studies showed that inflammatory response played a critical role in the pathogenesis of ALI $[3,4]$. LPS induces the release of inflammatory cytokine including TNF- $\alpha$ and IL-1 $\beta$, which exacerbate harmful immune responses $[5,6]$. Previous studies suggested that inhibition the release of inflammatory cytokines could treat ALI $[5,7]$. The production of inflammatory cytokines was regulated by NF- $\kappa B$ activation [8]. Inhibition of NF- $\kappa B$ activation could attenuate LPS-induced ALI [9].

Nrf2 is a nuclear factor that has been reported to control the expression of detoxifying enzymes [10]. Activation of Nrf2 could up-regulate the expression HO-1 [11]. Activation of Nrf2 signaling pathway could inhibit
NF- $\kappa B$ activation and inflammatory response [12]. Many herbal compounds protected against LPS-induced ALI by activation Nrf2 signaling pathway [13, 14]. Therefore, Nrf2 may be a promising target for treating lung injury. Recent studies showed that ALI was a kind of medium disease with mortality as high as $35 \sim 40 \%$ despite optimal supportive care, which caused great threat to people's lives and health $[15,16]$. Therefore, it is urgently to search for the safe and effective agent for the intervention of ALI.

In recent years, herbal drugs have become increasingly popular and their use is widespread [17]. A number of herbals and its natural compound have been reported to have anti-inflammatory effects $[18,19]$. Furthermore, many herbal medicine have the ability to attenuate LPS-induced ALI [20]. Gastrodin (GAS), a phenolic glucoside derived from Gastrodiaelata Blume, has been reported to have anti-inflammatory, anti-nociceptive, and anti-oxidative effects [21, 22]. Previous study showed that GAS attenuated the inflammatory response in $\mathrm{H} 9 \mathrm{c} 2$ 
cardiomyocytes [23]. Also, GAS was found to improve anti-oxidant and anti-inflammation activities and inhibit apoptosis pathway in cerebral ischemic damage [22]. Furthermore, it has been reported that GAS protects against $\mathrm{MPP}+$-induced oxidative stress in human dopaminergic cells [24]. GAS inhibits expression of inflammatory cytokines in LPS-stimulated microglia [25]. However, there have been no reports on the effects of GAS on LPSinduced acute lung injury. In this study we investigated the protective effects of GAS on LPS-induced ALI in mice.

\section{RESULTS}

\section{The effects of gastrodin on histopathological changes}

Lung tissues were collected at $12 \mathrm{~h}$ or $24 \mathrm{~h}$ after LPS treatment. Then the lung tissues were subjected to H\&E staining. Compared to the control group, LPS group showed severe histopathologic changes, including thicken of the alveolar wall, pulmonary congestion and alveolar wall thickness (Figure 1B). However, treatment with gastrodin $(15,30$ and $60 \mathrm{mg} / \mathrm{kg})$ significantly ameliorated LPS-induced histopathological changes (Figure 1C, 1D, 1E). Treatment with gastrodin $(60 \mathrm{mg} / \mathrm{kg}) 12 \mathrm{~h}$ after LPS treatment also significantly ameliorated LPS-induced histopathological changes (Figure 1F).

\section{The effects of gastrodin on MPO activity and lung wet to dry weight ratio}

MPO, a functional biomarker of neutrophils, was tested to assess the neutrophil accumulation in lung. We found that the MPO activity was significantly up-regulated in the LPS group compared with the control group. However, gastrodin treatment significant inhibited the activation of MPO (Figure 2A). We also detected the effects of gastrodin on lung wet to dry weight ratio. The results showed that lung wet to dry weight ratio was significantly up-regulated in the LPS group compared with the control group. However, gastrodin treatment significant inhibited LPS-induced lung wet to dry weight ratio (Figure 2B).

\section{Effects of gastrodin on lung function}

The effects of gastrodin on lung function were detected in this study. As shown in Figure 3, LPS significantly increased Raw and treatment of gastrodin inhibited LPS-induced Raw. Cdyn and PEF were decreased by LPS. Treatment of gastrodin significantly reversed the LPS-induced decrease of Cdyn and PEF.

\section{Effects of gastrodin on inflammatory cell count in the BALF}

Effects of gastrodin on inflammatory cell count in the BALF were measured in this study. As shown in Figure
4, the numbers of total cells, neutrophils, and macrophages increased significantly after LPS treatment. However, treatment of gastrodin significantly inhibited LPS-induced inflammatory cells infiltration in BALF (Figure 4).

\section{Gastrodin inhibits inflammatory cytokines release in $\mathrm{BALF}$}

Inflammatory cytokines has been reported to play critical roles in ALI. In the present study, the levels of TNF- $\alpha$, IL-6, and IL-1 $\beta$ in BALF were measured by ELISA assays. Compared with the control group, the levels of TNF- $\alpha$, IL- 6 , and IL- $1 \beta$ in BALF were increased in the LPS group. However, gastrodin treatment significantly inhibited the levels of inflammatory cytokines TNF- $\alpha$, IL6 , and IL-1 $\beta$ (Figure 5).

\section{Gastrodin inhibits NF- $\mathrm{KB}$ activation in LPS- induced ALI}

To investigate the anti-inflammatory mechanism of gastrodin, western blot analysis was applied to investigate the effects of gastrodin on LPS-induced NF- $\kappa$ B activation in lung tissues. The results showed thatLPS obviously

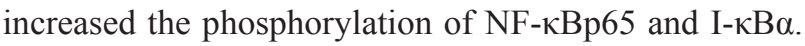
However, gastrodin treatment significantly inhibited the

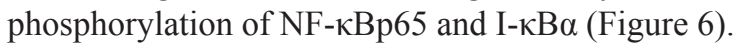

\section{The effects of gastrodin on Nrf2 and HO-1 expression}

The effects of gastrodin on Nrf2 signaling pathway were measured by western blot analysis. As shown in Figure 5, compared with the control group, the expression of Nrf2 and HO-1 increased in LPS-treated group. However, gastrodin dose-dependently increased the expression of $\mathrm{Nrf} 2$ and $\mathrm{HO}-1$ (Figure 7).

\section{DISCUSSION}

Acute lung injury (ALI) is a major clinical problem in the intensive care unit [26]. The mortality of ALI remains high in recent years. A large body of evidences showed that inhibition of inflammation may have protective effects against ALI [27]. In this study, we demonstrated that gastrodin protected against LPSinduced ALI by inhibiting inflammatory response.

LPS challenge leads to lung edema and inflammatory cells infiltration [28]. The infiltration of neutrophils results in the releasing of reactive oxygen species and inflammatory cytokines, which lead to lung injury. MPO was used as a marker for tissue neutrophils content [29]. Previous study showed that inhibition of neutrophils infiltration could attenuate LPS-induced lung injury [30]. In the present study, our results showed that gastrodin significantly inhibited LPS-induced inflammatory cells infiltration and MPO activity. Inflammatory cytokines are 


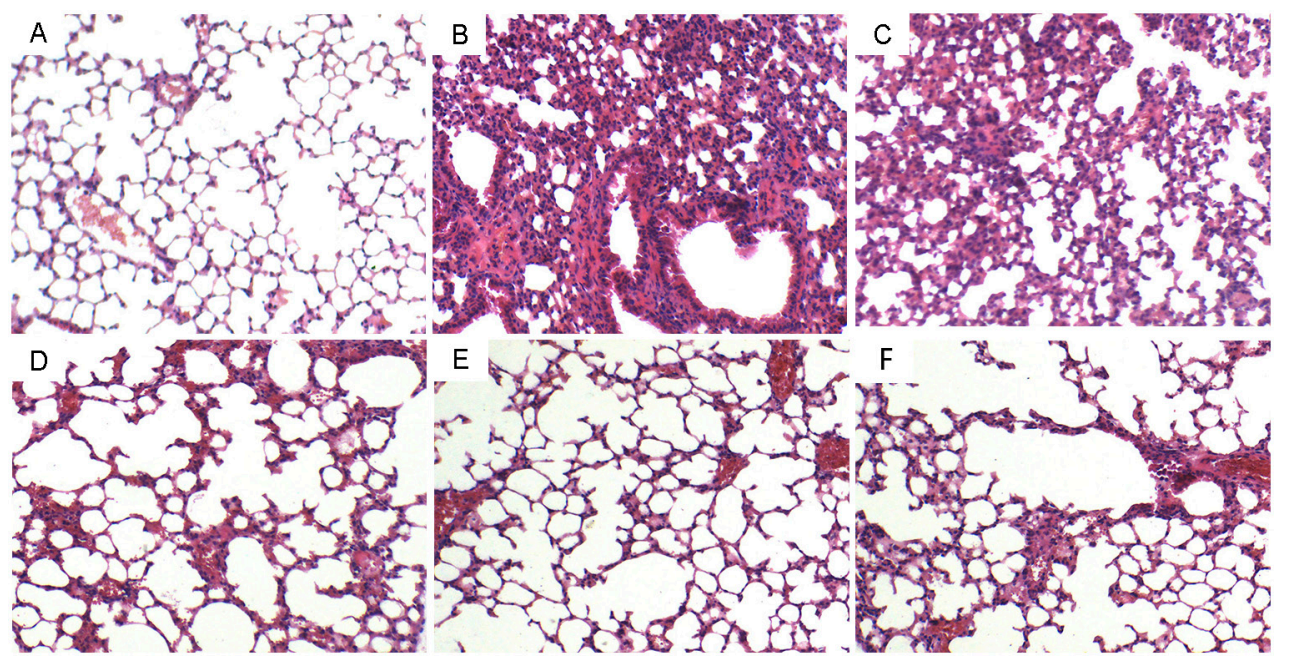

Figure 1: Effects of gastrodin on histopathological changes in lung tissues in LPS-induced ALI mice. Representative histological changes of lung obtained from mice of different groups. (A) Control group, (B) LPS group, (C) LPS + gastrodin (15 mg/kg) group, (D) LPS + gastrodin (30 mg/kg) group (E) LPS + gastrodin (60 mg/kg) group (F) LPS+gastrodin (60 mg/kg, $12 \mathrm{~h}$ later) (Hematoxylin and eosin staining, magnification $200 \times$ ).
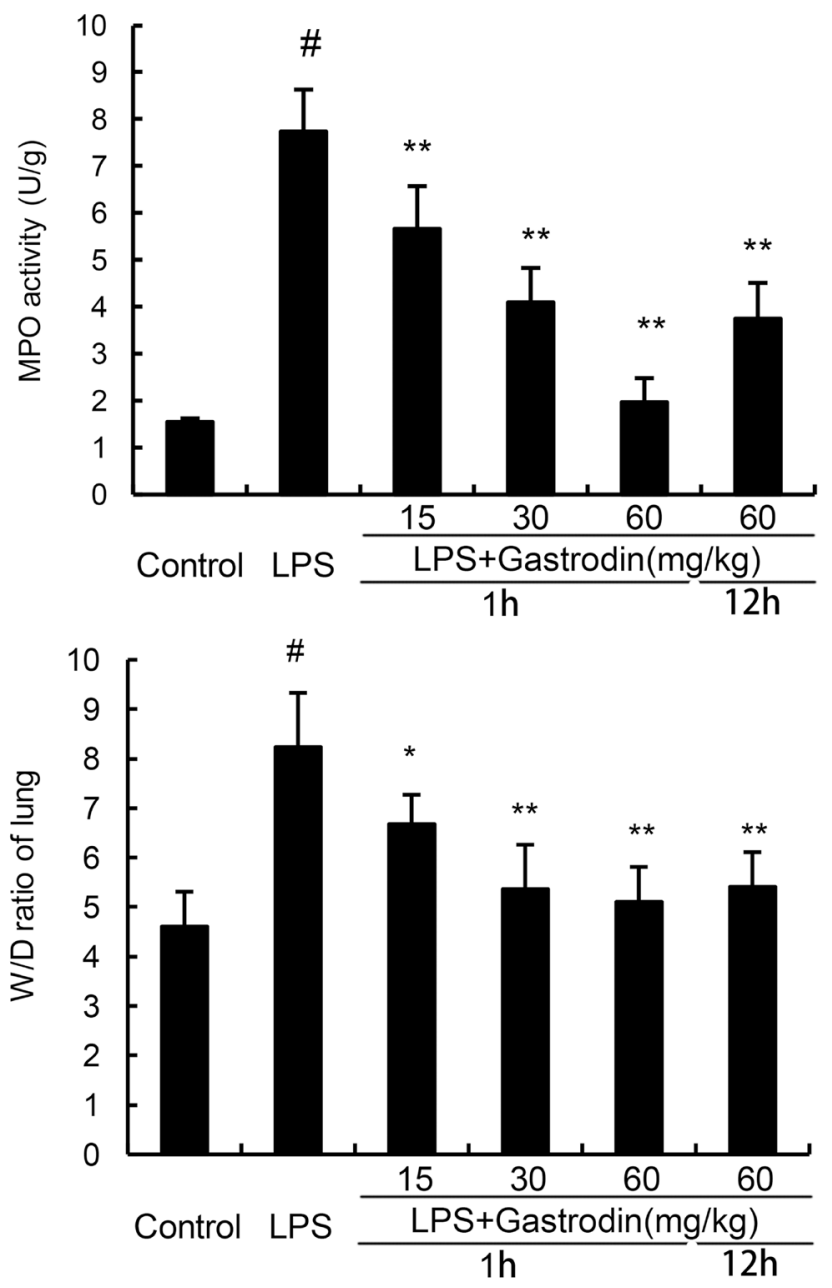

Figure 2: Effects of gastrodin on MPO activity in lung tissues and lung wet to dry weight ratio of LPS-induced ALI. The values presented are the mean $\pm \operatorname{SEM}\left(\mathrm{n}=12\right.$ in each group) of three independent experiments. $\mathrm{p} \#<0.01 \mathrm{vs}$. control group, $\mathrm{p}^{* *<0.01}$ vs. LPS group. 
known to be involved in the development of ALI [31]. In the model of LPS-induced ALI, inflammatory cytokines in BALF increased significantly [32]. These inflammatory cytokines TNF- $\alpha$, IL-1 $\beta$, and IL- 6 are associated with lung injury severity [33]. In this study, our results showed that gastrodin significantly inhibited LPS-induced inflammatory cytokines production. Furthermore, LPSinduced lung histopathological changes were attenuated by treatment of gastrodin. These results suggested that gastrodin had protective effects against LPS-induced ALI.

$\mathrm{NF}-\kappa \mathrm{B}$, an essential transcription factor, is a critical molecule in the regulation of inflammation [34, 35]. In normal conditions, $\mathrm{NF}-\kappa \mathrm{B}$ remains in the cytoplasm with
$\mathrm{I} \kappa \mathrm{B} \alpha$. Once stimulated by LPS, it will result in the release of $\mathrm{NF}-\kappa \mathrm{B}$ through phosphorylation and degradation of $\mathrm{I} \kappa \mathrm{B} \alpha$ [36]. Accumulating studies have provided strong evidence that NF- $\kappa \mathrm{B}$ was involved in the development of ALI [37]. Previous study showed that inhibition of NF- $\kappa B$ activation could attenuate the LPS-induced ALI [28]. To investigate the anti-inflammatory mechanism of gastrodin, the effects of gastrodin on NF- $\mathrm{BB}$ were measured. In the present study, we demonstrated that gastrodin significantly inhibited LPS-induced NF- $\kappa$ B activation. The results suggested that gastrodin inhibited LPS-induced inflammatory cytokines production via inhibiting NF- $\mathrm{NB}$ signaling pathway.
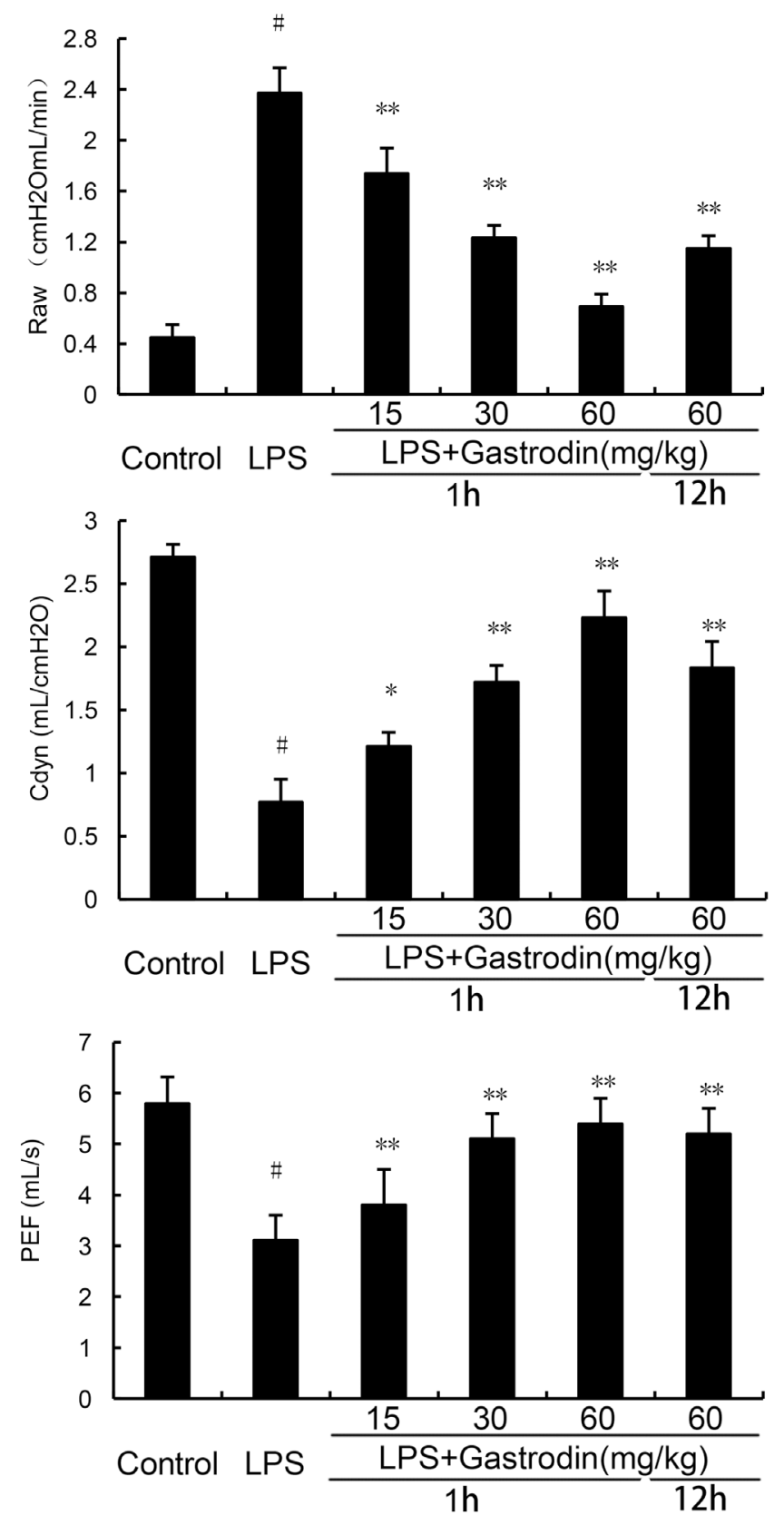

Figure 3: Effects of gastrodin on lung function. The values presented are the means $\pm \mathrm{SEM}$ of three independent experiments.

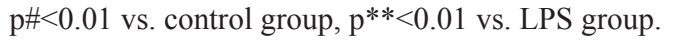


Nrf2 signaling pathway is a promising approach to protect against ALI, which regulates the oxidative and inflammatory responses $[38,39]$. Activating of Nrf2 could regulate the expression of antioxidant and antiinflammatory genes. Studies showed that many herbal compounds protected against LPS-induced lung injury by activating Nrf2 signaling pathway [40, 41]. Furthermore, it has been shown that Nrf2 has the ability to regulate the activation of NF- $\kappa \mathrm{B}$ [42]. It has been reported that transfection with HO-1 siRNA lead to the inhibition of $\mathrm{NF}-\kappa \mathrm{B}$ activation [43]. To further investigate the antiinflammatory mechanism of gastrodin, Nrf2 signaling
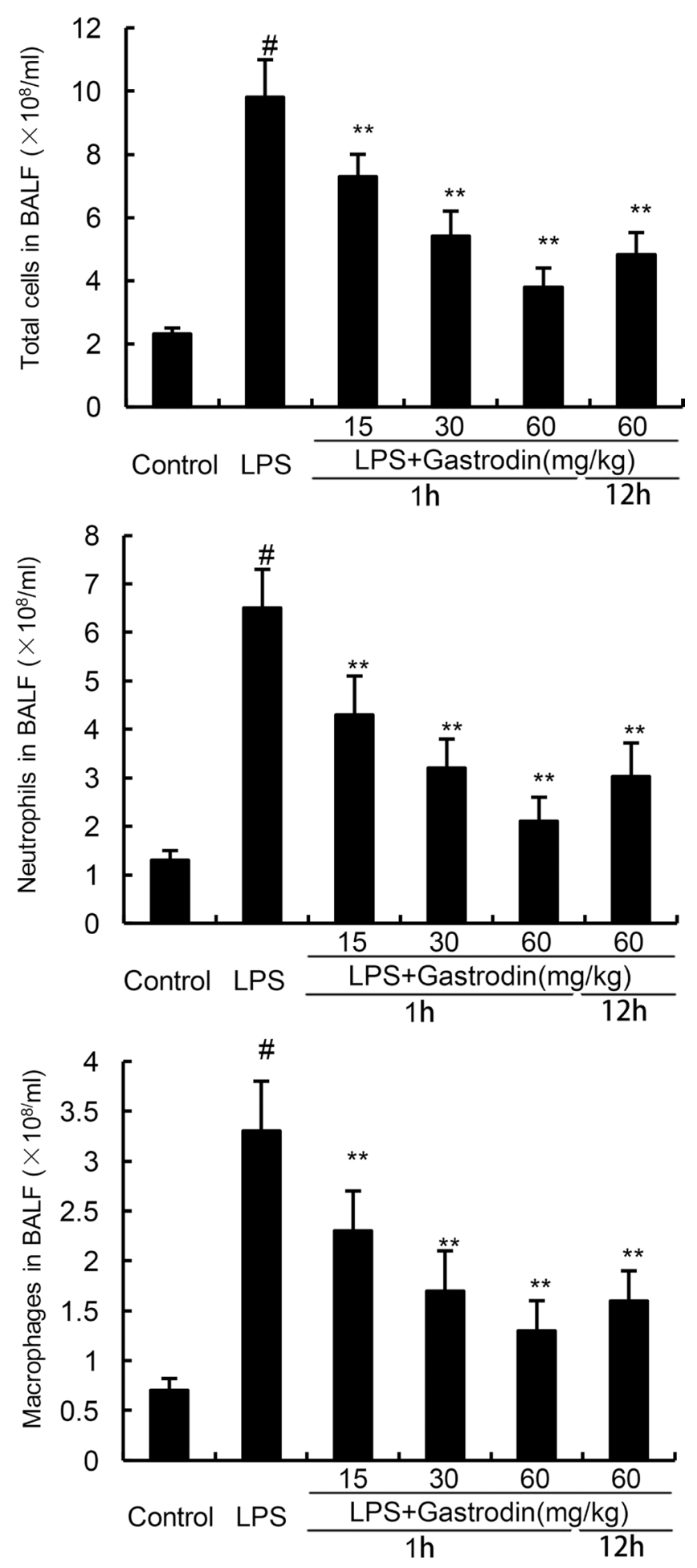

Figure 4: Effects of gastrodin on inflammatory cell count in the BALF of LPS-induced ALI mice. The values presented are mean $\pm \operatorname{SEM}\left(\mathrm{n}=12\right.$ in each group) of three independent experiments. $\mathrm{p} \#<0.01$ vs. control group, $\mathrm{p}^{*}<0.05$, $\mathrm{p}^{* *}<0.01$ vs. LPS group. 

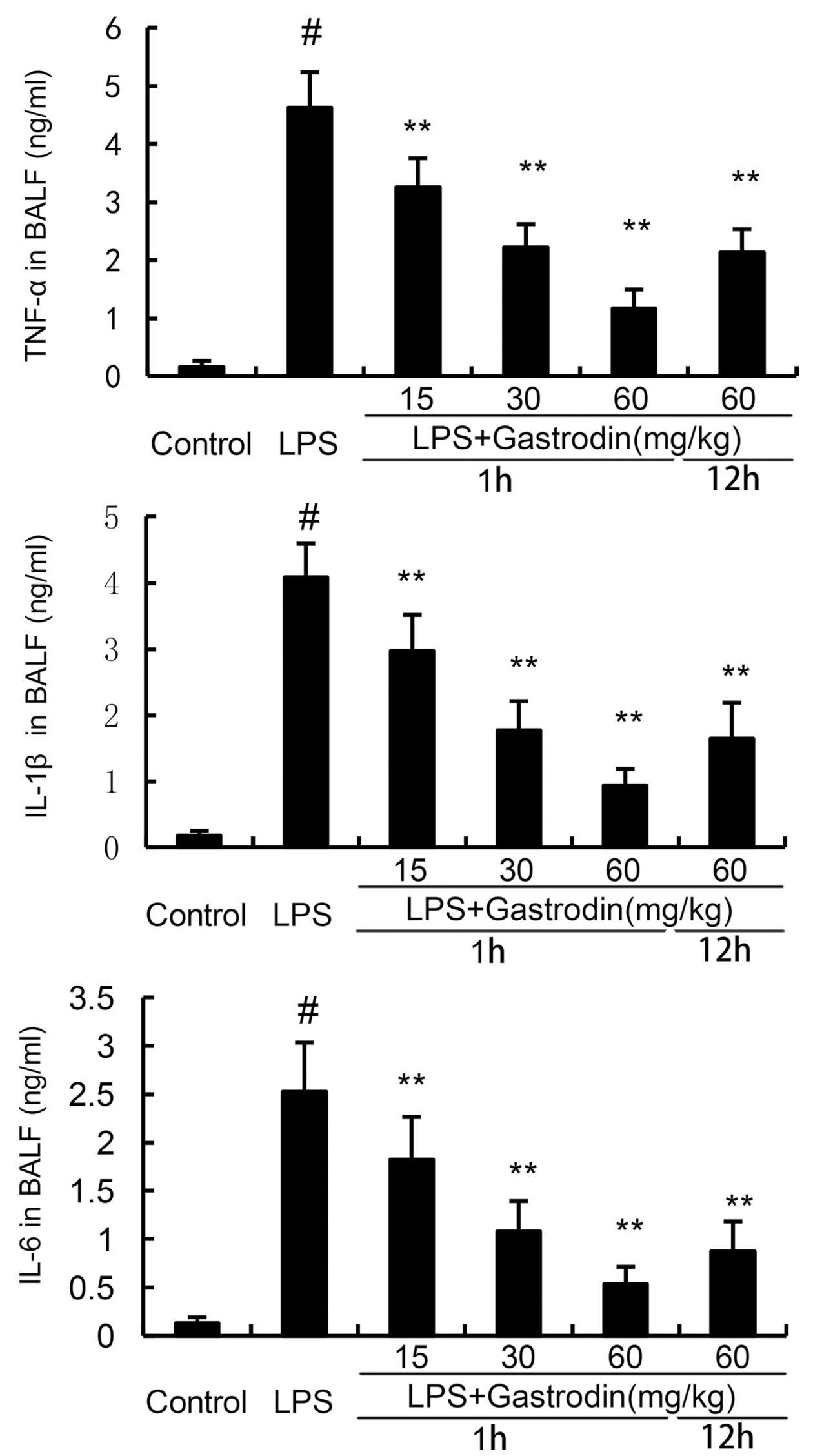

Figure 5: Effects of gastrodin on TNF- $\alpha$, IL-1B, and IL-6 production in the BALF of LPS-induced ALI mice. The values presented are mean $\pm \operatorname{SEM}\left(\mathrm{n}=12\right.$ in each group) of three independent experiments. $\mathrm{p} \#<0.01$ vs. control group, $\mathrm{p}^{* *}<0.01$ vs. LPS group. 

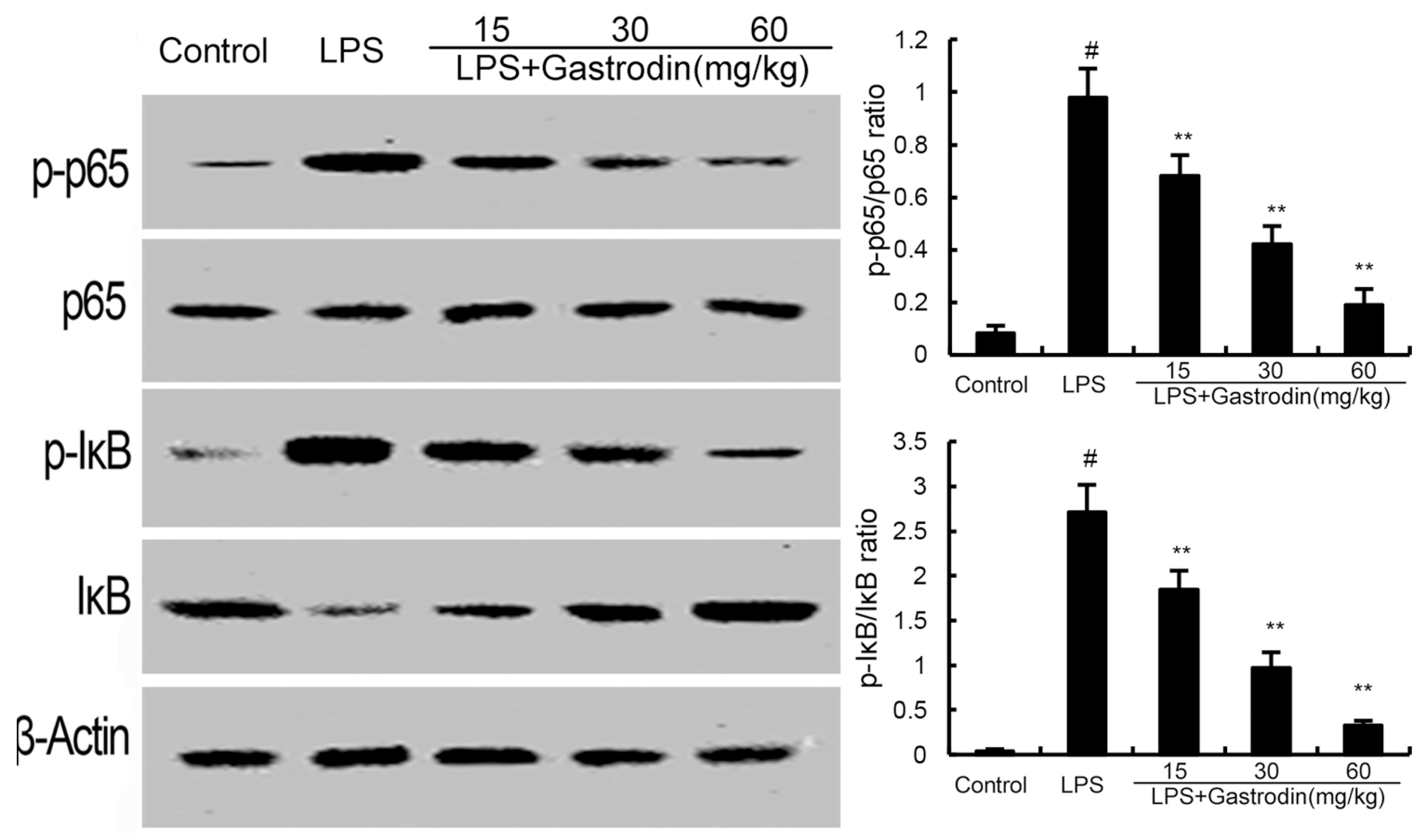

Figure 6: Gastrodin inhibits LPS-induced NF-кB activation. The values presented are the means \pm SEM of three independent

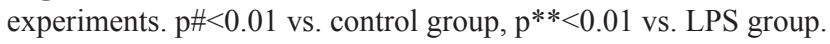

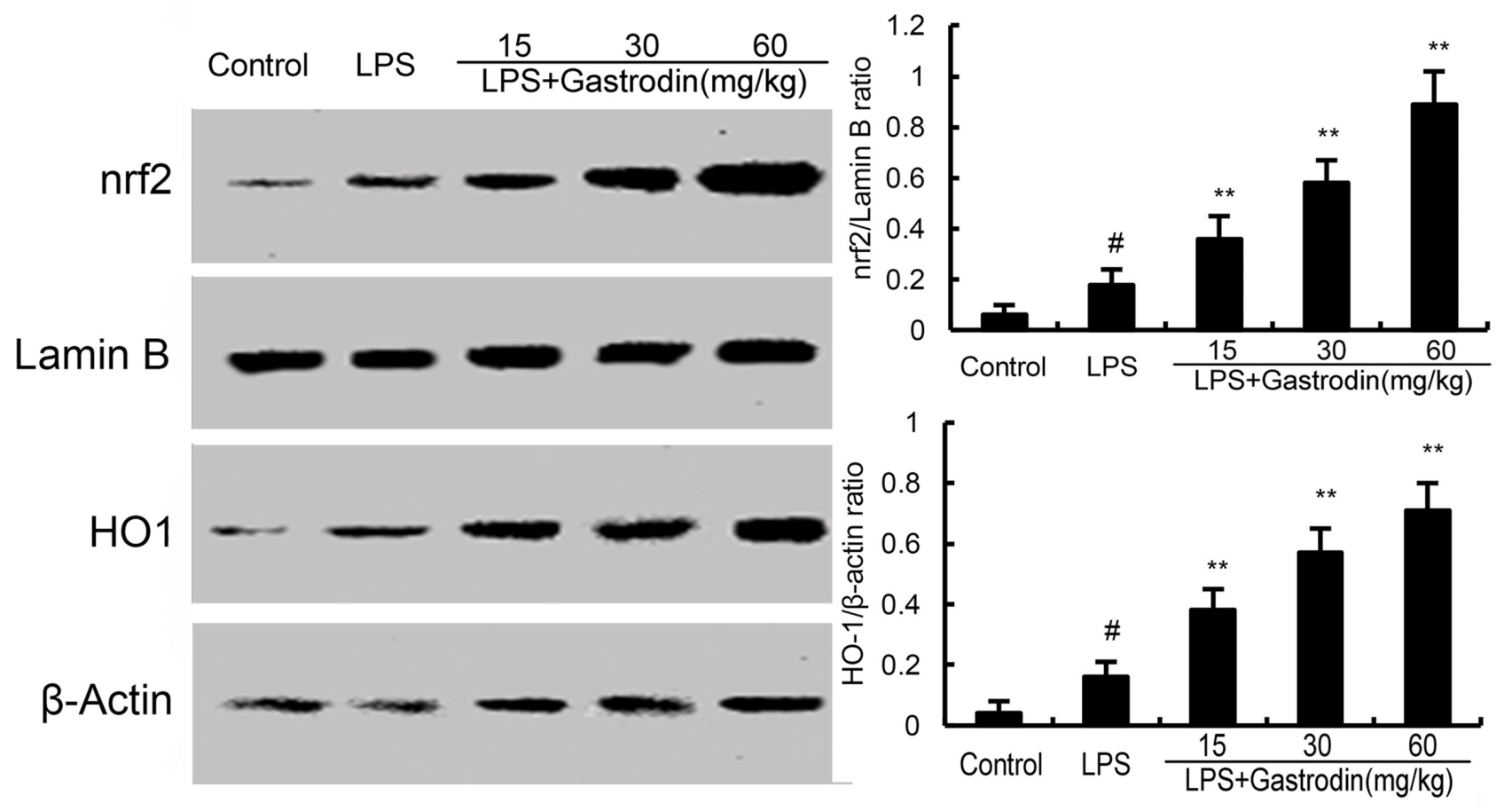

Figure 7: Effects of gastrodin on Nrf2 signaling pathway. The values presented are the means \pm SEM of three independent experiments. $\mathrm{p} \#<0.01$ vs. control group, $\mathrm{p}^{* *<0.01}$ vs. LPS group. 
pathway was detected in this study. We found that gastrodin dose-dependently increased the expression of Nrf2 and HO-1. These results demonstrated that gastrodin attenuated LPS-induced ALI by activating Nrf2 signaling pathway.

In conclusion, gastrodin treatment attenuates LPSinduced ALI in mice by inhibiting inflammatory cytokines production. The anti-inflammatory mechanism of gastrodin is through activating Nrf2, which subsequently inhibits LPS-induced NF- $\kappa$ B activation. Gastrodin might be an effective agent for the treatment of ALI.

\section{MATERIALS AND METHODS}

\section{Reagents}

Gastrodin (purity $>98 \%$ ) was purchased from SigmaAldrich (St. Louis, MO, USA). LPS was purchased from Sigma Chemical Co. (St. Louis, MO, USA). Antibodies for Nrf2, HO-1, NF- $\kappa \mathrm{B}$ p65, I $\kappa \mathrm{B} \alpha$, and $\beta$-actin were purchased from Santa Cruz Biotechnology (Autogen, Bioclear, UK). Mouse TNF- $\alpha$, IL-6 and IL-1 $\beta$ ELISA kits were obtained from R\&D (R\&D systems, Minneapolis, MN, USA). MPO detection kit was obtained from Nanjing Jiancheng Bioengineering institute (Nanjing, China).

\section{Acute lung injury model}

BALB/c mice, 6-8 weeks old, were purchased from Guangzhou Medical University (Guangzhou, China). The mice were given a standard diet and received water free access. All animal experiments were performed according to the Committee of Animal Experimentation of the Southwest medical university. Seventy-two mice were randomly divided into six groups and each group contains twelve mice: control group, LPS group, LPS + gastrodin $(15,30$ and $60 \mathrm{mg} / \mathrm{kg})$ groups, LPS + gastrodin $(60 \mathrm{mg} / \mathrm{kg})$ group (12 h later). $10 \mu \mathrm{g}$ of LPS was instilled intranasally (i.n.) in $50 \mu \mathrm{l}$ PBS to induce lung injury. Gastrodin $(15,30$ and $60 \mathrm{mg} / \mathrm{kg}$ ) was given $1 \mathrm{~h}$ or 12 after LPS treatment. Then, the mice were euthanized and the lung tissues and BALF were collected for the subsequent experiments.

\section{Histopathologic evaluation of lung tissues}

To detect histopathological changes, lung tissues were collected and fixed in $10 \%$ formalin for $12 \mathrm{~h}$, imbedded in paraffin and sliced. The sections were stained with hematoxylin and eosin (H\&E) staining. Finally, pathological changes in the lung tissues were examined under a light microscope.

\section{MPO assay}

12h after LPS challenge, the lung tissue samples were collected, for measuring MPO activity. MPO activity, represents the parenchymal infiltration of neutrophils, were examined by using the MPO determination kit (Nanjing Jiancheng Bioengineering institute, China) following the manufacturer's protocols.

\section{Lung wet-to-dry weight ratio}

The lung tissues of mice were excised, blotted dry, weighed to obtain the 'wet' weight. Then, the lung tissues were placed in an oven at $80^{\circ} \mathrm{C}$ for $72 \mathrm{~h}$ to obtain the 'dry' weight. The ratio of wet lung to dry lung was calculated to assess lung edema.

\section{Collection of BALF and cell counting}

The BALF were obtained by intratracheal instillation with $0.8 \mathrm{ml}$ cold PBS. Then the BALF samples were centrifuged and the supernatants were used to analysis the levels of cytokines. The cell pellets were suspended with PBS for cell counts. Neutrophils and macrophages in BALF were stained with the Kwik-Diff staining set (Thermo).

\section{Lung function assay}

Lung function including air resistance (Raw), lung dynamic compliance (Cdyn), and peak expiratory flow (PEF) was detected using small animal spirometer (PLY3211 system, Buxco Electronics, USA) as previously described [44].

\section{Inflammatory cytokines assay}

The BALF were harvested $12 \mathrm{~h}$ after LPS treatment and the levels of TNF- $\alpha$, IL- $1 \beta$, and IL- 6 in the BALF were measured by ELISA kits in accordance with the manufacturer's instructions (BioLegend, CA, USA).

\section{Western blot analysis}

The total proteins were extracted from lungs using Protein Extraction Kit according to the manufacturer's protocol (Beyotime, Haimen, China). Protein concentrations were measured using the BCA protein assay kit. The protein samples with equal amounts $(40 \mu \mathrm{g})$ of protein were separated on $12 \%$ SDS gel and transferred onto PVDF membranes. After blocking with $3 \%$ nonfat milk in TBST for $2 \mathrm{~h}$ at room temperature, the membrane was probed with the primary antibodies and HRP-conjugated secondary antibodies. The signals on the membranes were developed with the ECL Plus detection reagents (Thermo).

\section{Statistical analyses}

Comparison between groups were analyzed using one-way analysis of variance (ANOVA) and the twotailed Student's $t$ test. All data are presented as mean \pm 
SEM, with a $\mathrm{p}$ value $<0.05$ considered to be statistically significant.

\section{CONFLICTS OF INTEREST}

All authors declare that they have no conflicts of interest.

\section{FUNDING}

This work was supported by Education Department of Sichuan Province (13ZB0267); the Joint Research Fund for Luzhou Technology Bureau and Luzhou Medical College (14JC0181, 2013LZLY-J52); Science and Technology Agency of Sichuan (2014SZ0071).

\section{REFERENCES}

1. Butt Y, Kurdowska A, Allen TC. Acute Lung Injury: A Clinical and Molecular Review. Archives of pathology \& laboratory medicine. 2016; 140:345-350.

2. Mokra D, Kosutova P. Biomarkers in acute lung injury. Respir Physiol Neurobiol. 2015; 209:52-58.

3. Parsons PE, Eisner MD, Thompson BT, Matthay MA, Ancukiewicz M, Bernard GR, Wheeler AP, Network NARDSCT. Lower tidal volume ventilation and plasma cytokine markers of inflammation in patients with acute lung injury. Critical care medicine. 2005; 33:1-6; discussion 230-232.

4. Welbourn CR, Young Y. Endotoxin, septic shock and acute lung injury: neutrophils, macrophages and inflammatory mediators. The British journal of surgery. 1992; 79:998-1003.

5. Goodman RB, Pugin J, Lee JS, Matthay MA. Cytokinemediated inflammation in acute lung injury. Cytokine \& growth factor reviews. 2003; 14:523-535.

6. Strieter RM, Kunkel SL. Acute lung injury: the role of cytokines in the elicitation of neutrophils. Journal of investigative medicine. 1994; 42:640-651.

7. Su X, Wang L, Song Y, Bai C. Inhibition of inflammatory responses by ambroxol, a mucolytic agent, in a murine model of acute lung injury induced by lipopolysaccharide. Intensive care medicine. 2004; 30:133-140.

8. Tak PP, Firestein GS. NF-kappaB: a key role in inflammatory diseases. J Clin Invest. 2001; 107:7-11.

9. Everhart MB, Han W, Sherrill TP, Arutiunov M, Polosukhin VV, Burke JR, Sadikot RT, Christman JW, Yull FE, Blackwell TS. Duration and intensity of NF-kappaB activity determine the severity of endotoxin-induced acute lung injury. J Immunol. 2006; 176:4995-5005.

10. Jaiswal AK. Nrf2 signaling in coordinated activation of antioxidant gene expression. Free radical biology \& medicine. 2004; 36:1199-1207.

11. Vargas MR, Pehar M, Cassina P, Martinez-Palma L, Thompson JA, Beckman JS, Barbeito L. Fibroblast growth factor-1 induces heme oxygenase-1 via nuclear factor erythroid 2-related factor 2 (Nrf2) in spinal cord astrocytes: consequences for motor neuron survival. J Biol Chem. $2005 ; 280: 25571-25579$.

12. Chen XL, Dodd G, Thomas S, Zhang X, Wasserman MA, Rovin BH, Kunsch C. Activation of Nrf2/ARE pathway protects endothelial cells from oxidant injury and inhibits inflammatory gene expression. American journal of physiology Heart and circulatory physiology. 2006; 290:H1862-1870.

13. Jiang W, Luo F, Lu Q, Liu J, Li P, Wang X, Fu Y, Hao K, Yan T, Ding X. The protective effect of Trillin LPS-induced acute lung injury by the regulations of inflammation and oxidative state. Chem Biol Interact. 2016; 243:127-134.

14. Sun CY, Xu LQ, Zhang ZB, Chen CH, Huang YZ, Su ZQ, Guo HZ, Chen XY, Zhang X, Liu YH, Chen JN, Lai XP, $\mathrm{Li}$ YC, Su ZR. Protective effects of pogostone against LPS-induced acute lung injury in mice via regulation of Keap1-Nrf2/NF-kappaB signaling pathways. Int Immunopharmacol. 2016; 32:55-61.

15. Gotts JE, Matthay MA. Treating ARDS: new hope for a tough problem. The Lancet Respiratory medicine. 2014; 2:84-85.

16. Ferguson ND, Fan E, Camporota L, Antonelli M, Anzueto A, Beale R, Brochard L, Brower R, Esteban A, Gattinoni L, Rhodes A, Slutsky AS, Vincent JL, et al. The Berlin definition of ARDS: an expanded rationale, justification, and supplementary material. Intensive care medicine. 2012; 38:1573-1582.

17. Stickel F, Schuppan D. Herbal medicine in the treatment of liver diseases. Digestive and liver disease. 2007; 39:293-304.

18. Miller MJ, Vergnolle N, McKnight W, Musah RA, Davison CA, Trentacosti AM, Thompson JH, Sandoval M, Wallace JL. Inhibition of neurogenic inflammation by the Amazonian herbal medicine sangre de grado. The Journal of investigative dermatology. 2001; 117:725-730.

19. He DY, Dai SM. Anti-inflammatory and immunomodulatory effects of paeonia lactiflora pall., a traditional chinese herbal medicine. Frontiers in pharmacology. 2011; 2:10.

20. Chen X, Yang X, Liu T, Guan M, Feng X, Dong W, Chu X, Liu J, Tian X, Ci X, Li H, Wei J, Deng Y, et al. Kaempferol regulates MAPKs and NF-kappaB signaling pathways to attenuate LPS-induced acute lung injury in mice. Int Immunopharmacol. 2012; 14:209-216.

21. Wang XL, Xing GH, Hong B, Li XM, Zou Y, Zhang XJ, Dong MX. Gastrodin prevents motor deficits and oxidative stress in the MPTP mouse model of Parkinson's disease: involvement of ERK1/2-Nrf2 signaling pathway. Life sciences. 2014; 114:77-85.

22. Peng Z, Wang S, Chen G, Cai M, Liu R, Deng J, Liu J, Zhang T, Tan Q, Hai C. Gastrodin alleviates cerebral ischemic damage in mice by improving anti-oxidant and anti-inflammation activities and inhibiting apoptosis pathway. Neurochem Res. 2015; 40:661-673. 
23. Yang P, Han Y, Gui L, Sun J, Chen YL, Song R, Guo JZ, Xie YN, Lu D, Sun L. Gastrodin attenuation of the inflammatory response in H9c2 cardiomyocytes involves inhibition of NF-kappaB and MAPKs activation via the phosphatidylinositol 3-kinase signaling. Biochemical pharmacology. 2013; 85:1124-1133.

24. Jiang G, Hu Y, Liu L, Cai J, Peng C, Li Q. Gastrodin protects against $\mathrm{MPP}(+)$-induced oxidative stress by up regulates heme oxygenase-1 expression through p38 MAPK/Nrf2 pathway in human dopaminergic cells. Neurochemistry international. 2014; 75:79-88.

25. Dai JN, Zong Y, Zhong LM, Li YM, Zhang W, Bian LG, Ai QL, Liu YD, Sun J, Lu D. Gastrodin Inhibits Expression of Inducible NO Synthase, Cyclooxygenase-2 and Proinflammatory Cytokines in Cultured LPS-Stimulated Microglia via MAPK Pathways. Plos One. 2011; 6:e21891.

26. Brun-Buisson C, Minelli C, Bertolini G, Brazzi L, Pimentel J, Lewandowski K, Bion J, Romand JA, Villar J, Thorsteinsson A, Damas P, Armaganidis A, Lemaire FO, Grp AS. Epidemiology and outcome of acute lung injury in European intensive care units - Results from the ALIVE study. Intensive care medicine. 2004; 30:524-524.

27. Pontes-Arruda A, DeMichele S, Seth A, Singer P. The Use of an Inflammation-Modulating Diet in Patients With Acute Lung Injury or Acute Respiratory Distress Syndrome: A Meta-Analysis of Outcome Data. Jpen-Parenter Enter. 2008; 32:596-605.

28. Shen W, Gan J, Xu S, Jiang G, Wu H. Penehyclidine hydrochloride attenuates LPS-induced acute lung injury involvement of NF-kappaB pathway. Pharmacological research. 2009; 60:296-302.

29. Bradley PP, Priebat DA, Christensen RD, Rothstein G. Measurement of cutaneous inflammation: estimation of neutrophil content with an enzyme marker. The Journal of investigative dermatology. 1982; 78:206-209.

30. Sakamaki F, Ishizaka A, Urano T, Sayama K, Nakamura H, Terashima T, Waki Y, Tasaka S, Hasegawa N, Sato K, Nakagawa N, Obata T, Kanazawa M. Effect of a specific neutrophil elastase inhibitor, ONO-5046, on endotoxininduced acute lung injury. American journal of respiratory and critical care medicine. 1996; 153:391-397.

31. Goodman RB, Strieter RM, Martin DP, Steinberg KP, Milberg JA, Maunder RJ, Kunkel SL, Walz A, Hudson LD, Martin TR. Inflammatory cytokines in patients with persistence of the acute respiratory distress syndrome. American journal of respiratory and critical care medicine. 1996; 154:602-611.

32. Wei D, Huang Z. Anti-inflammatory effects of triptolide in LPS-induced acute lung injury in mice. Inflammation. 2014; 37:1307-1316.

33. Lee IT, Yang CM. Inflammatory signalings involved in airway and pulmonary diseases. Mediators of inflammation. 2013; 2013:791231.
34. Fu Y, Hu X, Cao Y, Zhang Z, Zhang N. Saikosaponin a inhibits lipopolysaccharide-oxidative stress and inflammation in Human umbilical vein endothelial cells via preventing TLR4 translocation into lipid rafts. Free radical biology \& medicine. 2015; 89:777-785.

35. Fu Y, Tian Y, Wei Z, Liu H, Song X, Liu W, Zhang W, Wang W, Cao Y, Zhang N. Liver X receptor agonist prevents LPSinduced mastitis in mice. Int Immunopharmacol. 2014; 22:379-383.

36. Wang J, Guo C, Wei Z, He X, Kou J, Zhou E, Yang Z, Fu Y. Morin suppresses inflammatory cytokine expression by downregulation of nuclear factor-kappaB and mitogenactivated protein kinase (MAPK) signaling pathways in lipopolysaccharide-stimulated primary bovine mammary epithelial cells. Journal of dairy science. 2016; 99:3016-3022.

37. Yang R, Yang L, Shen X, Cheng W, Zhao B, Ali KH, Qian Z, Ji H. Suppression of NF-kappaB pathway by crocetin contributes to attenuation of lipopolysaccharideinduced acute lung injury in mice. European journal of pharmacology. 2012; 674:391-396.

38. Marzec JM, Christie JD, Reddy SP, Jedlicka AE, Vuong H, Lanken PN, Aplenc R, Yamamoto T, Yamamoto M, Cho HY, Kleeberger SR. Functional polymorphisms in the transcription factor NRF2 in humans increase the risk of acute lung injury. FASEB journal. 2007; 21:2237-2246.

39. Chan KM, Kan YW. Nrf2 is essential for protection against acute pulmonary injury in mice. P Natl Acad Sci USA. 1999; 96:12731-12736.

40. Yao WF, Luo GJ, Zhu GS, Chi XJ, Zhang AL, Xia ZY, Hei ZQ. Propofol Activation of the Nrf2 Pathway Is Associated with Amelioration of Acute Lung Injury in a Rat Liver Transplantation Model. Oxid Med Cell Longev. 2014; 2014:258567.

41. Kim KH, Han W, Sadikot R, Christman J, Breyer R, Chan J, Urade Y, Oh YK, Blackwell T, Joo M. Prostaglandin D2 ameliorates acute lung inflammation by activating Nrf2 independent of 15-deoxy-Delta 12,14-PGJ2. J Immunol. 2013; 190.

42. Cuadrado A, Martin-Moldes Z, Ye JP, Lastres-Becker I. Transcription Factors NRF2 and NF-kappa B Are Coordinated Effectors of the Rho Family, GTP-binding Protein RAC1 during Inflammation. J Biol Chem. 2014; 289:15244-15258.

43. Lee YM, Auh QS, Lee DW, Kim JY, Jung HJ, Lee SH, Kim EC. Involvement of Nrf2-mediated upregulation of heme oxygenase- 1 in mollugin-induced growth inhibition and apoptosis in human oral cancer cells. BioMed research international. 2013; 2013:210604.

44. Yao H, Chung S, Hwang JW, Rajendrasozhan S, Sundar IK, Dean DA, McBurney MW, Guarente L, Gu W, Ronty M, Kinnula VL, Rahman I. SIRT1 protects against emphysema via FOXO3-mediated reduction of premature senescence in mice. J Clin Invest. 2012; 122:2032-2045. 